\title{
Bruxismo nocturno y síndrome de dolor miofascial.
}

\author{
Sleep bruxism and myofascial pain syndrome.
}

\author{
Jaime Alejandri-Gamboa,* Estefany Yessenia Gómez-Moreno*
}

\section{RESUMEN}

El bruxismo es un factor iniciador y coadyuvante de alteraciones de la región temporomandibular, entre las que se incluye el síndrome de dolor miofascial. Sin embargo, en los diversos estudios realizados en la literatura científica no hay homogeneidad en los métodos diagnósticos, en la clasificación de bruxismo o en las alteraciones temporomandibulares, lo que no permite asegurar que existe una relación causa-efecto entre el bruxismo y los diferentes desórdenes temporomandibulares. Para intentar aclarar la relación entre el bruxismo y el síndrome de dolor miofascial se realizó una revisión sistemática de la literatura sobre estudios que hayan buscado identificar la asociación entre las dos condiciones. Los estudios que han sugerido una relación causal entre bruxismo nocturno y dolor miofascial han utilizado métodos diagnósticos variables, apoyándose, principalmente, en encuestas de corte transversal, lo cual ha limitado la posibilidad de lograr conclusiones firmes; mientras que aquéllos que usan métodos objetivos, como polisomnografía, no han encontrado una concluyente relación causal.

Palabras clave: Dolor miofascial, bruxismo nocturno, dolor facial, desórdenes temporomandibulares.

\section{ABSTRACT}

Bruxism is an initiating and coadjuvant factor of temporomandibular disorders, including myofascial pain syndrome. However, in the various studies conducted in the scientific literature, there is a lack of consistency in diagnostic methods, the classification of bruxism, or temporomandibular disorders, which has prevented to ensure that there are a cause and effect relationship between bruxism and the different temporomandibular disorders. To try to clarify the relationship between bruxism and myofascial pain syndrome, a systematic review of the literature was carried out on studies that have sought an association about these two conditions. Studies that have found a causal relationship between nocturnal bruxism and myofascial pain have used variable diagnostic methods, relying mainly on surveys, which has limited the possibility of reaching firm conclusions; while those who used objective methods, such as polysomnography, have not found a conclusive causal relationship.

Keywords: Myofascial pain, sleep bruxism, facial pain, temporomandibular disorders.

\section{INTRODUCCIÓN}

$\mathrm{E}$ I bruxismo es una alteración parafuncional neuromuscular que se manifiesta con movimientos rítmicos de la mandíbula (RJM [Rhytmic Jaw Movement]), que se reflejan en apretamiento o rechinamiento dental, con o sin desgaste. El bruxismo se clasifica de acuerdo con sus manifestaciones clínicas en bruxismo diurno y nocturno. El bruxismo nocturno (BN) se caracteriza por apretamiento o rechinamiento dental durante el sueño, acompañado

\footnotetext{
* Residente del Postgrado de Prostodoncia e Implantología, Facultad de Odontología de la Universidad De La Salle Bajío.
}

Recibido: 13 Agosto 2019.

Aceptado para publicación: 13 Julio 2020. de microdespertares y excitación neuronal. ${ }^{1}$ Su etiología y fisiopatología no son bien comprendidas.

Durante décadas se ha considerado un iniciador o promotor de trastornos temporomandibulares, en particular del dolor miofascial. Es difícil de evaluar y diagnosticar sin el uso de estudios de polisomnografía (PSG), ofrecidos por laboratorios especializados. ${ }^{2}$ La polisomnografía consiste en la toma de registros de electroencefalograma, electrocardiograma, electromiograma, electrooculograma y respiración durante el sueño. ${ }^{3}$ Se utiliza para encontrar alteraciones de la respiración durante el sueño, parasomnias, narcolepsia y desórdenes del ritmo circadiano. Requiere pasar dos noches en una clínica de sueño, la primera para habituarse al ambiente y la segunda para la evaluación. Para el diagnóstico del bruxismo se realizan electromiogramas de los músculos maseteros y temporales y electroencefalograma, junto con grabaciones audiovisuales. Los criterios encontrados para confirmar presencia 
de bruxismo nocturno son: más de cuatro episodios de actividad muscular masticatoria rítmica (RMMA [Rhytmic Masticatory Muscle Activity]), más de 25 ráfagas en el electromiograma (EMG), y por lo menos un episodio con sonido de rechinamiento, todos dentro de un periodo de una hora de sueño. ${ }^{4}$ Los estudios científicos que usan este método diagnóstico son raros debido a su costo elevado, acceso limitado y el tiempo prolongado que requieren. Los estudios de polisomnografía en México tienen un costo alrededor de 10 mil pesos y los estudios simplificados pueden costar la mitad. Estos se pueden realizar en hospitales o clínicas del sueño.

Aun hoy, la noción de que el bruxismo juega un papel significativo en la patogénesis de las alteraciones temporomandibulares (ATM), combinándose con alteraciones oclusales, es muy común. ${ }^{5}$ Sin embargo, se ha demostrado que la etiología de las ATM no sólo se origina por alteraciones oclusales, también es multifactorial e involucra cambios neuronales, alteraciones sistémicas, traumáticas y psicológicas, todas ellas teniendo como un factor común a la hiperfuncionalidad muscular; dejando en un plano secundario a las alteraciones de tipo oclusal. ${ }^{6}$

El síndrome de dolor miofascial (SDM) es uno de los desórdenes temporomandibulares (TMD) más comunes. Clínicamente se identifican focos hiperirritables llamados puntos gatillo, habitualmente en zonas de tensión muscular repetida. El dolor que generan puede ser sordo, pulsátil o muy agudo, que se agrava con la función. Su etiología no se ha determinado con certeza. Se cree que puede deberse a cargas musculares ligeras por periodos largos de tiempo o a cargas elevadas repetidas. ${ }^{7}$ La mayor parte de los puntos gatillo mejora o se resuelve en algunas semanas sin necesidad de tratamiento específico. ${ }^{8}$ Muestra una mayor prevalencia en mujeres.

Los puntos gatillo pueden ser activos o estar latentes. Los puntos activos tienen patrones de dolor referidos a sitios cercanos fuera del músculo afectado, como las áreas auriculotemporal, intraoral, supraorbitaria y maxilar. ${ }^{8}$ Los puntos gatillo de la cara y el cuello son una fuente común de cefaleas, se ubican en los músculos maseteros, pterigoideos, trapecios y esternocleidomastoideos. Los puntos gatillo no se pueden identificar por pruebas de laboratorio o imagenología, y su valoración electrofisiológica es complicada; por lo cual su valoración clínica está confinada a la palpación, buscando nódulos firmes localizados sensibles, ${ }^{9}$ lo que lo hace un método muy subjetivo y poco reproducible. ${ }^{10}$ El SDM puede existir sin alteraciones de la articulación temporomandibular.

Ante la disparidad de criterios diagnósticos y la falta de certeza de su origen, el tratamiento del SDM se ha enfocado en disminuir su sintomatología y controlar el apretamiento del bruxismo para disminuir sus efectos deletéreos secundarios. ${ }^{11-13}$ El manejo del SDM se puede realizar con fármacos, acupuntura, fisioterapia, electroterapia y modificaciones posturales. A menudo se usa una combinación o secuencia dependiendo de cada paciente. ${ }^{14}$

Algunos pacientes experimentan el dolor más intenso en las mañanas (21\%) asociado con BN, o en las tardes (79\%) por apretamiento diurno, mientras que otros no tienen un patrón fijo. Su duración promedio es alrededor de seis horas por día y su frecuencia puede ser diaria o episódica, pudiendo haber días sin dolor. ${ }^{15}$ El dolor se puede agravar durante la función mandibular, con episodios de dolor espontáneo; el dolor provocado por la función puede ser el principal motivo de consulta del paciente. ${ }^{16}$

El dolor miofascial y el dolor provocado por el bruxismo pueden afectar la calidad de vida del paciente. El dolor provocado puede disminuir la eficacia de masticación, reducir la calidad del sueño y crear signos de depresión. ${ }^{17}$

\section{MATERIAL Y MÉTODOS}

Se realizó una búsqueda sistemática de artículos bajo la pregunta ¿el bruxismo es causante de dolor miofascial?, en los buscadores PubMed y EBSCOhost: sleep bruxism, bruxism, TMD. Temporomandibular disorders, myofascial pain syndrome; en un periodo de 1994 hasta diciembre de 2016. Se revisaron los artículos que incluyeran definición, etiología y tratamientos para síndrome de dolor miofascial y la relación que se ha encontrado con bruxismo nocturno. Los artículos fueron leídos por el autor principal y fueron seleccionados de acuerdo con su contenido. Se excluyeron artículos que no usaran PSG o electromiografía (EMG) para diagnosticar el bruxismo.

\section{RESULTADOS}

En la Tabla 1 se muestran los artículos analizados e incluidos para la elaboración de la revisión del tema.

Los datos encontrados indican que el BN probablemente no es un condicionante de alteraciones temporomandibulares. Al usar métodos de evaluación más objetivo de bruxismo nocturno, como la PSG, no se encuentra una relación causa-efecto clara. Varios estudios han encontrado que los pacientes con dolor miofascial son más propensos que los controles a mostrar bruxismo nocturno. ${ }^{18-20}$ Sólo algunos, basados en autorreportes y entrevista al paciente, encontraron una relación positiva 
Tabla 1: Artículos elegidos para la revisión.

\begin{tabular}{|c|c|c|c|}
\hline Autor & Tipo de estudio & Muestra & Conclusiones \\
\hline $\begin{array}{r}\text { Raphael KG } \\
\text { et al. } 2012\end{array}$ & Autorreporte entrevistas & 124 pacientes & La mayoría de los participantes del caso no presentaron SB \\
\hline $\begin{array}{l}\text { Türp JC \& } \\
\quad \text { Schindler H. } 2012\end{array}$ & Artículo de revisión & 2,342 pacientes & $\begin{array}{l}\text { Se llegó a la conclusión de que la oclusión desempeñaba sólo } \\
\text { un papel menor junto con los signos y síntomas de TMD }\end{array}$ \\
\hline $\begin{array}{l}\text { Quek } \\
\text { et al. } 2014\end{array}$ & Estudio comparativo & 200 pacientes & $\begin{array}{l}\text { MNB proporcionó un efecto terapéutico inmediato para el trata- } \\
\text { miento del dolor miofascial }\end{array}$ \\
\hline $\begin{array}{l}\text { Lavigne GJ } \\
\text { et al. } 1995\end{array}$ & $\begin{array}{l}\text { Análisis de regresión } \\
\text { logística }\end{array}$ & 36 pacientes & $\begin{array}{l}\text { En los pacientes asintomáticos no existe presencia de signos o } \\
\text { síntomas asociados al bruxismo, en los pacientes con bruxismo hay } \\
\text { presencia de ellos }\end{array}$ \\
\hline $\begin{array}{l}\text { Manfredini D } \\
\text { et al. } 2013\end{array}$ & Revisión sistemática & 35 publicaciones & $\begin{array}{l}\text { Las actividades de bruxismo no estaban relacionadas con el sexo, y } \\
\text { se describió una disminución con la edad en las personas mayores }\end{array}$ \\
\hline $\begin{array}{l}\text { Manfredini D, } \\
\text { Lobbezoo } \\
\text { F. } 2010\end{array}$ & Revisión sistemática & 46 artículos & $\begin{array}{l}\text { Se encuentra una relación menor en los estudios basados en méto- } \\
\text { dos más cuantitativos y específicos para diagnosticar la asociación } \\
\text { entre el bruxismo y TMD. En estudios basados en el autoinforme o } \\
\text { diagnóstico clínico se obtiene una asociación positiva }\end{array}$ \\
\hline $\begin{array}{l}\text { Lucas } \mathrm{N} \\
\quad \text { et al. } 2009\end{array}$ & Revisión sistemática & 9 estudios & $\begin{array}{l}\text { No se encontró un estudio hasta la fecha que reporte la fiabilidad } \\
\text { del diagnóstico de puntos gatillo, con los criterios que se proponen } \\
\text { en esta revisión }\end{array}$ \\
\hline $\begin{array}{l}\text { Rossetti LN } \\
\text { et al. } 2008\end{array}$ & Estudio comparativo & $\begin{array}{l}\text { Estudio } \\
\text { comparativo }\end{array}$ & $\begin{array}{l}\text { Se obtuvo que SB está significativamente asociado con SDM. } \\
\text { Que SB representa un factor de riesgo bajo para SDM }\end{array}$ \\
\hline $\begin{array}{l}\text { Nunes, Rossetti, } \\
\text { et al. } 2008\end{array}$ & Estudio comparativo & 26 pacientes & $\begin{array}{l}\text { No se encontró asociación entre bruxismo y TMD, ni entre el } \\
\text { bruxismo y el dolor a la palpación }\end{array}$ \\
\hline $\begin{array}{l}\text { Raphael KG } \\
\text { et al. } 2013\end{array}$ & Estudio comparativo & 170 pacientes & $\begin{array}{l}\text { EMG mayor en los casos de TMD que en los de control. Mayor } \\
\text { actividad en los músculos masticatorios durante el sueño en los } \\
\text { casos de TMD }\end{array}$ \\
\hline
\end{tabular}

$\mathrm{SB}=$ sleeping bruxism; $\mathrm{TMD}$ = temporomandibulares; $\mathrm{MNB}=$ Mental Nerve Block; $\mathrm{SDM}$ = síndrome de dolor miofascial; $\mathrm{EMG}=$ electromiografía

entre las dos condiciones. Por esto no se puede concluir que la hiperactividad de los músculos masticatorios del bruxismo nocturno sea una explicación para el dolor miofascial persistente, o viceversa.

Estudios que utilizaron PSG han sugerido que el BN y el dolor miofascial probablemente tendrían etiologías no relacionadas. Un estudio encontró que el dolor originado por el bruxismo era mayor en las mañanas, mientras que el dolor miofascial era mayor durante las tardes, y que no todos los pacientes con bruxismo presentan dolor. ${ }^{21} \mathrm{Se}$ encontró sólo un trabajo apoyado también en PSG, en éste encuentran una asociación positiva entre el bruxismo nocturno y el dolor miofascial, considerando al $\mathrm{BN}$ un factor de riesgo para dolor miofascial. ${ }^{22}$

En general, los estudios basados en PSG no encuentran una relación entre bruxismo nocturno y dolor miofascial.
Utilizando electromiografía (EMG) se ha reportado que no hay diferencia entre pacientes con o sin dolor miofascial en la actividad muscular, ${ }^{23}$ mientras que otro estudio concluyó que la actividad muscular intensa del bruxismo es inversamente proporcional al número de episodios de RMMA por hora de sueño, indicando que en pacientes con mayor dolor había menos actividad de los músculos masticatorios. ${ }^{24}$

Desde hace 50 años se ha considerado al bruxismo como un factor predominante en la etiología, preservación y severidad de los desórdenes temporomandibulares, ${ }^{25}$ pero el papel que juega es controversial. No está claro si el apretamiento o rechinamiento provocados por la hiperactividad muscular del $\mathrm{BN}$ son factores causantes del dolor o si es el espasmo muscular el que lleva a una contracción reactiva. 
Algunos autores no han encontrado una relación directa y constante entre estos factores, ya que sólo algunos pacientes con bruxismo presentan dolor, ${ }^{23}$ y una posible explicación es el fenómeno de adaptación muscular; se ha observado que aun en presencia de una hiperactividad muscular funcional o patológica, la musculatura se puede adaptar rápido y volverse más resistente a ejercicios repetidos. ${ }^{24,25}$

Algunos pacientes experimentan el dolor más intenso en las mañanas (21\%) asociado a BN, o en las tardes (79\%) por apretamiento diurno, mientras que otros no tienen un patrón fijo, su frecuencia puede ser diaria o episódica, y pueden pasar días sin dolor. El dolor se puede agravar durante la función mandibular, con episodios de dolor espontáneo; el dolor provocado por la función suele ser el principal motivo de consulta del paciente. ${ }^{16}$

\section{DISCUSIÓN}

La relación de bruxismo con diversas disfunciones de la ATM ha sido muy controversial, debido a los métodos de diagnóstico y el conocimiento de la etiología de este tipo de desórdenes. Los estudios basados en cuestionarios o autorreportes muestran una relación positiva entre bruxismo y dolor miofascial, pero tienen un elevado potencial de error y confusión por el método de diagnóstico.

La mayoría de los estudios que han encontrado que existe una relación entre los desórdenes temporomandibulares y el bruxismo están basados en autorreportes, ya que es la forma más simple de reunir muestras grandes, en vez de PSG, que requiere costo, acceso y tiempo, además de que no hay estandarización en el diagnóstico de desórdenes temporomandibulares.

Los estudios de PSG han encontrado menor relación con el dolor miofascial. Si la hiperactividad muscular es contribuyente del dolor en pacientes con dolor miofascial y bruxismo, estos pacientes deberían experimentar dolor masticatorio reducido cuando la hiperactividad muscular masticatoria se reduzca. Para conducir una prueba clínica que explore las relaciones entre hiperactividad muscular y dolor de los músculos de la masticación se requiere una intervención que reduzca la hiperactividad de forma confiable.

\section{Recomendaciones clínicas}

Los pacientes con bruxismo y dolor miofascial pueden reducir el dolor con ayuda de guardas oclusales, que disminuyen la actividad muscular anormal y producen un balance neuromuscular, pero no eliminan el apreta- miento ni rechinamiento dental. ${ }^{24}$ Los guardas oclusales tienen la ventaja de ser simples, no invasivos y de menor costo que otros tratamientos, además de necesitar poco tiempo para ser fabricados. Al colocar el guarda oclusal, se da el número ideal de contactos masticatorios y guías de desoclusión, bloquea interferencias y permite que los músculos se relajen y su actividad se estabilice. Son mucho más seguros que usar tratamiento farmacológico a largo plazo. Cuando hay dolor muscular, el contacto interdental no funcional aumenta hasta cuatro veces, ${ }^{26}$ por lo que un guarda también puede ayudar a proteger los dientes de las fuerzas oclusales.

Se debe cuidar tener un diagnóstico preciso para SDM y BN, aunque no siempre sea posible realizar PSG. En un dolor difícil de localizar el origen puede tener otra causa. Los pacientes deben ser educados acerca del SDM y $\mathrm{BN}$. Los probables mecanismos de dolor les deben ser explicados de forma simple, además de informar que el dolor muscular no es causado por daño a los tejidos y que no representa peligro. Hay que determinar si existen situaciones que provoquen estrés en cada paciente, ya que se ha encontrado que es posible que alteraciones psicológicas exacerben al bruxismo y al SDM. Asimismo, se debe informar de la importancia del descanso y del ejercicio, ya que ayudan a reducir la sobrecarga muscular, y tal vez algunos pacientes tendrán que ser referidos con profesionales de la salud mental. En algunos pacientes, el dolor provocado por el SDM puede requerir tratamiento farmacológico sistémico o directamente sobre los puntos gatillo; sin embargo, el ejercicio y la reducción de estrés debería incluirse en el tratamiento de todos los pacientes que presenten SDM. ${ }^{27}$

Al dar tratamiento farmacológico se debe considerar que el SDM tiene componentes musculares y nerviosos. Hay pocos estudios sobre el uso de AINEs para el SDM, pero se han usado comúnmente en su tratamiento, y se ha encontrado mayor éxito con la aplicación de AINEs tópicos sobre los puntos gatillo. Se debe informar al paciente de posibles efectos secundarios, además de realizar monitoreo en pacientes en quienes estos fármacos ayudan al control del dolor, y descontinuarlos en pacientes en los que no son efectivos. Las benzodiacepinas, como el clonazepam, han encontrado reducción significante de los síntomas, pero sus efectos secundarios pueden provocar riesgos durante el tratamiento. ${ }^{28}$

Los relajantes musculares son benéficos en el tratamiento del síndrome de dolor miofascial, ya que se origina de espasmos musculares o tensión muscular. La tizanidina, que inhibe la liberación de neurotransmisores excitadores, es uno de los más utilizados, y se debe ad- 
ministrar durante la noche debido a su efecto sedante. ${ }^{29}$ Se debe iniciar con una dosis de 2-4 mg en las noches $y$, dependiendo de la severidad del dolor, aumentar a tres dosis por día, sin alcanzar un máximo de $8 \mathrm{mg} .{ }^{28}$ Debe evitar administrarse en pacientes que toman alfa agonistas, antiarrítmicos y fluoroquinolonas.

Los AINEs pueden reducir el dolor, pero tienen menor eficiencia que los relajantes musculares.

En caso del bruxismo diurno, es posible educar al paciente para que intente dejar de apretar al ser consciente de que lo está haciendo, y esto puede ser tan eficiente como un guarda oclusal, pero dado que el rechinamiento en el bruxismo nocturno es una actividad completamente inconsciente, es más conveniente proteger el sistema estomatognático con el uso de un guarda, ya que la propiocepción disminuye en la noche, y no se alcanzan a percibir fuerzas que se detectarían en estado consciente.

\section{CONCLUSIONES}

Es muy difícil establecer de manera segura una relación entre dolor miofascial y el bruxismo nocturno. Ambas condiciones pueden coexistir de manera independiente, sin que su etiología esté ligada.

Basándose solamente en estudios con métodos diagnósticos objetivos, no se puede encontrar una verdadera asociación entre el bruxismo y el dolor miofascial, por lo que no es posible establecer su relación con completa certeza, pero su cantidad reducida limita las conclusiones que es posible alcanzar.

Asimismo, es necesario educar al paciente sobre el tratamiento, la importancia del sueño, reconocer factores psicológicos como ansiedad, depresión o estrés que puedan exacerbar la aparición de puntos gatillo y bruxismo, y de ser necesario, remitirlos a profesionales de la salud mental.

En el ambiente clínico también se debe considerar la posible dificultad de llevar a cabo estudios de polisomnografía, ya sea debido a disponibilidad, tiempo o costo. En estos casos es posible que el autorreporte o entrevista sea un método apropiado para evaluar la presencia de bruxismo, siempre y cuando se tomen en cuenta las deficiencias que pueden originarse por las limitaciones de este método.

Por último, no se debe tratar al bruxismo y al dolor miofascial como una sola entidad, sino como dos problemas separados en un mismo paciente, y es necesario indagar en los antecedentes sistémicos de los sujetos más allá de los hallazgos locales para dilucidar las posibles causas de ambas condiciones.

\section{AGRADECIMIENTOS}

Se agradece por su contribución al doctor Juan Manuel Guizar Mendoza, catedrático de la materia de investigación del postgrado en prostodoncia e implantología de la Facultad de Odontología de la Universidad de La Salle Bajío, el doctor Robert Mosqueda Martínez, catedrático del postgrado en prostodoncia e implantología, y la doctora María Teresa Zermeño Loredo, coordinadora del postgrado en prostodoncia e implantología de la Facultad de Odontología de la Universidad de La Salle Bajío.

\section{BIBLIOGRAFÍA}

1. Lobbezoo F, Ahlberg J, Glaros AG et al. Bruxism defined and graded: an international consensus. J Oral Rehabil. 2013; 40 (1): 2-4.

2. Lavigne GJ, Rompré PH, Montplaisir JY. Sleep bruxism: validity of clinical research diagnostic criteria in a controlled polysomnographic study. J Dent Res. 1996; 75 (1): 546-552.

3. Patil SP. What every clinician should know about polysomnography. Respir Care. 2010; 55 (9): 1179-1195.

4. Rompré PH, Daigle-Landry D, Guitard F, Montplaisir JY, Lavigne G). Identification of a sleep bruxism subgroup with a higher risk of pain. J Dent Res. 2007; 86 (9): 837-842.

5. Raphael KG, Sirois DA, Janal MN et al. Sleep bruxism and myofascial temporomandibular disorders: a laboratory-based polysomnographic investigation. J Am Dent Assoc. 2012; 143 (11): 1223-1231.

6. Türp JC, Schindler H. The dental occlusion as a suspected cause for TMDs: epidemiological and etiological considerations. J Oral Rehabil. 2012; 39 (7): 502-512.

7. Bron C, Dommerholt JD. Etiology of myofascial trigger points. Curr Pain Headache Rep. 2012; 16 (5): 439-444.

8. Bennett R. Myofascial pain syndromes and their evaluation. Best Pract Res Clin Rheumatol. 2007; 21 (3): 427-445.

9. Wright EF. Referred craniofacial pain patterns in patients with temporomandibular disorder. J Am Dent Assoc. 2000; 131 (9): 1307-1315.

10. Lucas N, Macaskill P, Irwig L, Moran R, Bogduk N. Reliability of physical examination for diagnosis of myofascial trigger points: a systematic review of the literature. Clin J Pain. 2009; 25 (1): 80-89.

11. Manfredini D, Lobbezoo F. Relationship between bruxism and temporomandibular disorders: a systematic review of literature from 1998 to 2008. Oral Surg Oral Med Oral Pathol Oral Radiol Endod. 2010; 109 (6): e26-e50.

12. Lobbezoo F, Lavigne GJ. Do bruxism and temporomandibular disorders have a cause-and-effect relationship? J Orofac Pain. 1997; 11 (1): 15-23.

13. Svensson P, Jadidi F, Arima T, Baad-Hansen L, Sessle BJ. Relationships between craniofacial pain and bruxism. J Oral Rehabil. 2008; 35 (7): 524-547.

14. Borg-Stein J, laccarino MA. Myofascial pain syndrome treatments. Phys Med Rehabil Clin N Am. 2014; 25 (2): 357-374.

15. Schwartz L. A temporomandibular joint pain dysfunction syndrome. J Chronic Dis. 1956; 3: 284-293.

16. Fernandes G, Franco AL, Siqueira JT, Gonçalves DA, Camparis CM. Sleep bruxism increases the risk for painful temporomandibular disorder, depression and non-specific physical symptoms. J Oral Rehabil. 2012; 39 (7): 538-544. 
17. Dao TT, Lund JP, Lavigne GJ. Comparison of pain and quality of life in bruxers and patients with myofascial pain of the masticatory muscles. J Orofac Pain. 1994; 8 (4): 350-356.

18. Raphael KG, Janal MN, Sirois DA et al. Validity of self-reported sleep bruxism among myofascial temporomandibular disorder patients and controls. J Oral Rehabil. 2015; 42 (10): 751-758.

19. Yachida W, Castrillon EE, Baad-Hansen L et al. Craniofacial pain and jaw-muscle activity during sleep. J Dent Res. 2012; 91 (6): 562-567.

20. Raphael KG, Janal MN, Sirois DA et al. Masticatory muscle sleep background electromyographic activity is elevated in myofascial temporomandibular disorder patients. J Oral Rehabil. 2013; 40 (12): 883-891.

21. Michelotti A, lodice G, Vollaro S, Steenks MH, Farella M. Evaluation of the short-term effectiveness of education versus an occlusal splint for the treatment of myofascial pain of the jaw muscles. J Am Dent Assoc. 2012; 143 (1): 47-53.

22. Rossetti LM, Pereira de Araujo Cdos R, Rossetti PH, Conti PC. Association between rhythmic masticatory muscle activity during sleep and masticatory myofascial pain: a polysomnographic study. J Orofac Pain. 2008; 22 (3): 190-200.

23. Benoliel R, Sharav Y. Masticatory myofascial pain, and tension-type and chronic daily headache. Orofacial Pain and Headache. 2008; 1 (7): 109-148

24. Raphael KG, Marbach JJ, Klausner JJ, Teaford MF, Fischoff DK. Is bruxism severity a predictor of oral splint efficacy in patients with myofascial face pain? J Oral Rehabil. 2003; 30 (1): 17-29.
25. Rossetti LM, Rossetti PH, Conti PC, de Araujo Cdos R. Association between sleep bruxism and temporomandibular disorders: a polysomnographic pilot study. Cranio. 2008; 26 (1): 16-24.

26. Chen CY, Palla S, Erni S, Sieber M, Gallo LM. Nonfunctional tooth contact in healthy controls and patients with myogenous facial pain. J Orofac Pain. 2007; 21 (3): 185-193.

27. Velly AM, Gornitsky M, Philippe P. Contributing factors to chronic myofascial pain: a case-control study. Pain. 2003; 104 (3): 491-499.

28. Fishbain DA, Cutler RB, Rosomoff HL, Rosomoff RS. Clonazepam open clinical treatment trial for myofascial syndrome associated chronic pain. Pain Med. 2000; 1 (4): 332-339.

29. Malanga GA, Gwynn MW, Smith R, Miller D. Tizanidine is effective in the treatment of myofascial pain syndrome. Pain Physician. 2002; 5 (4): 422-432.

\section{Correspondencia:}

\section{Jaime Alejandri Gamboa}

Av. Universidad Núm. 602,

Col. Lomas del Sol, 37150

León, Guanajuato, México.

E-mail: drjaimealejandri@gmail.com 